\title{
IDENTIFICACIÓN MOLECULAR DE Aspergillus fumigatus AISLADOS DE PACIENTES CON ASPERGILOSIS INVASIVA
}

\author{
Vilma Béjar ${ }^{1,2, a, d}$, Freddy Villanueva ${ }^{2,3, b}$, Segundo R. León ${ }^{1,4, b, e}$, José M. Guevara-Granados ${ }^{1,2, c}$, Alfonzo Uribe $^{6, e, f}$, \\ German Vergaray ${ }^{5, a, g}$, Ana Cuadra ${ }^{1,2, c}$, Iván Sabogal ${ }^{1,2, c}$
}

\begin{abstract}
RESUMEN
El objetivo del estudio fue identificar molecularmente cepas de aspergillus aislados de pacientes con aspergilosis invasiva (Al), que fueron tipificadas primariamente como Aspergillus fumigatus sensu lato por métodos fenotípicos convencionales. Se trabajó con 20 cepas de la micoteca de la sección de micología del Instituto de Medicina Tropical "Daniel A. Carrión". Para obtener el ADN fúngico se emplearon las técnicas de choque térmico, tratamiento enzimático y columnas de silica-gel; y se almacenó a $-20{ }^{\circ} \mathrm{C}$ para conservarlo. En el procedimiento de la reacción en cadena de la polimerasa en tiempo real (qPCR) se incluyeron primers marcados con fluorocromo, los cuales amplificaron las secuencias específicas de $A$. fumigatus. La fluorescencia se midió con el termociclador al final de la fase de hibridación de cada ciclo. Se identificó molecularmente que sólo el $50 \%$ de las cepas estudiadas pertenecen a la especie Aspergillus fumigatus sensu stricto.
\end{abstract}

Palabras clave: Aspergillus fumigatus; Aspergilosis Pulmonar Invasiva; Tipificación Molecular; Perú (fuente: DeCS BIREME).

\section{MOLECULAR IDENTIFICATION OF Aspergillus fumigatus ISOLATED FROM PATIENTS WITH INVASIVE ASPERGILLOSIS}

\begin{abstract}
The objective of the study was to identify molecularly-isolated strains of Aspergillus from patients with invasive aspergillosis (IA); these strains were primarily typed as Aspergillus fumigatus sensu lato by conventional phenotypic methods. We worked with 20 strains from the mycology section of the Institute of Tropical Medicine "Daniel A. Carrión." To obtain the fungal DNA, thermal shock, enzymatic treatment, and silica gel column techniques were used; and it was stored at $-20^{\circ} \mathrm{C}$ to preserve it. The real-time polymerase chain reaction (qPCR) procedure included fluorochrome-labeled primers, which amplified the specific sequences of A. fumigatus. Fluorescence was measured with the thermocycler at the end of the hybridization phase of each cycle. It was molecularly-identified that only $50 \%$ of the strains studied belong to the species Aspergillus fumigatus sensu stricto.
\end{abstract}

Keywords: Aspergillus fumigatus; Invasive Pulmonary Aspergillosis; Molecular typing; Peru (source: MeSH NLM).

\section{INTRODUCCIÓN}

La aspergilosis invasiva (Al) es causada por varias especies de micromicetos del género Aspergillus, en la mayoría de los casos reportados, el agente etiológico fue Aspergillus fumigatus ${ }^{(1)}$. El diagnóstico de la Al involucra el análisis microbiológico, el cual se efectúa mediante el estudio de las características fenotípicas del micromiceto; de esta manera se identifica a las principales especies de Aspergillus causantes de Al (2). Sin embargo, en los últimos años se ha evidenciado la existencia de cepas resistentes a los antimicóticos y de características moleculares diferentes, en aislamientos considerados morfológicamente como $A$. fumigatus, lo cual ha puesto en duda la identificación

\footnotetext{
Instituto de Medicina Tropical “Daniel A. Carrión”. Lima, Perú

Departamento Académico de Microbiología Médica, Universidad Nacional Mayor de San Marcos. Lima, Perú

Instituto Nacional de Enfermedades Neoplásicas. Lima, Perú

Universidad Privada San Juan Bautista. Lima, Perú

Instituto de Investigación de Ciencias Biológicas "Antonio Raimondi”. Lima, Perú

Hospital Nacional Dos de Mayo. Lima, Perú

Biólogo, ${ }^{\mathrm{b}}$ tecnólogo médico, ${ }^{\mathrm{c}}$ médico cirujano, ${ }^{\mathrm{d}}$ magister en Salud Pública, ${ }^{\mathrm{e}}$ magister en Enfermedades Infecciosas y Tropicales, ${ }^{\mathrm{f}}$ doctor en Medicina, ${ }^{\mathrm{g}}$ doctor en Ciencias Biológicas.

Recibido: 22/01/2018 Aprobado: 20/02/2019 En línea: 19/03/2019
}

Citar como: Béjar V, Villanueva F, León SR, Guevara-Granados JM, Uribe A, Vergaray G, et al. Identificación molecular de Aspergillus fumigatus aislados de pacientes con aspergilosis invasiva. Rev Peru Med Exp Salud Publica. 2019;36(1):81-6. doi:10.17843/rpmesp.2019.361.3403. 
fenotípica convencional, motivando la aplicación de técnicas moleculares, lo que ha conducido a un cambio significativo en la taxonomía de la especie Aspergillus fumigatus.

Actualmente, se considera como Aspergillus fumigatus sensu lato a las especies tipificadas morfológicamente y como Aspergillus fumigatus sensu stricto a las tipificadas mediante estudios moleculares ${ }^{(3)}$. Asimismo, se ha creado la sección Fumigati ${ }^{(4,5)}$, que incluye a $A$. fumigatus, que consta de 25 especies, ocho en estado anomorfo (Aspergillus) y 17 en estado teleomorfo (Neorsartorya) ${ }^{(6,7)}$. Debido a ello, mediante técnicas moleculares se han diferenciado especies morfológicamente similares a $A$. fumigatus, creándose nuevas especies como $A$. lentulus, y $A$. viridinutans las cuales han sido incluidas en dicha sección.

Aplicando técnicas moleculares, se ha demostrado hasta en $6 \%$ que las $\mathrm{Al}$ en humanos son causadas por especies del complejo Aspergillus fumigatus sensu lato diferentes a Aspergillus fumigatus sensu stricto ${ }^{(8)}$. En aislamientos de otras fuentes, los resultados son variados, por ejemplo, de 146 cepas de Aspergillus fumigatus sensu lato aisladas principalmente de humanos, suelo y animales, se comprobó que 140 (95,8\%) eran $A$. fumigatus sensu stricto ${ }^{(5)}$; en otro estudio en el que se analizaron 17 muestras de suelo se aislaron 23 cepas de aspergillus sección Fumigati y se demostró que sólo nueve $(34,13 \%)$ eran $A$. fumigatus sensu stricto $^{(9)}$.

En las últimas décadas, se ha producido un incremento significativo de Al. Aun cuando han aumentado las opciones terapéuticas, las tasas de mortalidad se mantienen elevadas ${ }^{(10)}$. Diversos estudios, han revelado un incremento de la resistencia de $A$. fumigatus a los antifúngicos ${ }^{(11)}$. Asimismo, las especies de aspergillus de la sección Fumigati han presentado patrones variables de sensibilidad a los antifúngicos que incluyen resistencia intrínseca y sensibilidades reducidas ${ }^{(12)}$. Actualmente, no se dispone de estudios sobre la identificación molecular de las especies de aspergillus que vienen afectando a la población peruana, por lo que, no se puede establecer un esquema terapéutico adecuado ni un programa apropiado de control y vigilancia epidemiológica.

El objetivo del presente estudio fue identificar molecularmente cepas de aspergillus aisladas de pacientes con Al, que fueron tipificadas como Aspergillus fumigatus sensu lato por métodos fenotípicos.

\section{EL ESTUDIO}

\section{DISEÑO DE ESTUDIO Y POBLACIÓN}

Se realizó un estudio descriptivo y observacional. Se trabajó con 20 cepas de Aspergillus fumigatus sensu lato (identificados morfológicamente) de la micoteca de la Sección de Micología del Instituto de Medicina

\section{MENSAJES CLAVE}

Motivación para realizar el estudio. En las últimas décadas, se ha producido un incremento significativo de las aspergilosis invasiva (AI); el agente causante principal es Aspergillus fumigatus, el cual es identificado con técnicas fenotípicas por métodos convencionales. En algunos casos, la infección ha sido resistente al tratamiento, lo cual puede deberse a que no siempre se trata del mismo micromiceto. Ello ha conducido a realizar un estudio que permita una identificación más precisa del patógeno aplicando técnicas moleculares.

Principales hallazgos. Sólo el 50\% de los aislamientos clínicos de AI, previamente tipificados por su fenotipo como A. fumigatus sensu lato fueron confirmados por procedimientos moleculares como A. fumigatus sensu stricto.

Implicancias. Identificar específicamente la etiología de la $\mathrm{AI}$, permitirá establecer un esquema terapéutico adecuado. Asimismo, brindará información para que la autoridad de salud implemente medidas de control y vigilancia.

Tropical «Daniel A. Carrión» (IMT/DAC) de la Facultad de Medicina de la Universidad Nacional Mayor de San Marcos (UNMSM); las cepas fueron aisladas de muestras seriadas de esputo (14), de biopsia de tejido pulmonar (4) y de aspirado bronquial (2), de pacientes con diagnóstico clínico de Al procedentes de dos hospitales públicos de Lima (Hospital Nacional Dos de Mayo y Hospital Nacional Daniel Alcides Carrión). El estudio molecular se realizó en el laboratorio de la Sección de Epidemiología Molecular y Genética del IMT/DAC.

\section{IDENTIFICACIÓN FENOTÍPICA DE ASPERGILLUS FUMIGATUS SENSU LATO}

Las cepas de $A$. fumigatus sensu lato fueron estudiadas morfológicamente según la técnica de Klich y Pitt ${ }^{(2)}$. Cada cepa se sembró en dos placas de Petri que contenían agar Czápek con extracto de levadura, se incubaron a $25{ }^{\circ} \mathrm{C}$ y $37{ }^{\circ} \mathrm{C}$ durante siete días. Para la identificación macroscópica, se tomó en consideración, el diámetro, el color y el aspecto de la colonia, además, se tomó en cuenta la difusión de pigmento en el medio de cultivo. Para el estudio microscópico, se utilizaron microcultivos coloreados con azul de lactofenol y se tomó en cuenta la disposición de las métulas o fiálides sobre la vesícula, la forma y el diámetro de la vesícula, y la forma y color de los conidios.

\section{IDENTIFICACIÓN MOLECULAR DE A. FUMIGATUS SENSU STRICTO POR PCR EN TIEMPO REAL}

Las cepas de Aspergillus fumigatus sensu lato fueron reaisladas en agar papa dextrosa, 72 horas antes del procedimiento de extracción de ADN. Se cosecharon conidios e hifas mediante el raspado del micelio $\left(1 \mathrm{~cm}^{2}\right)$, se lavaron con $8 \mathrm{~mL}$ de solución salina estéril y se centrifugaron 
a $3000 \mathrm{~g}$ por cinco minutos. Se descartó el sobrenadante y se transfirió $400 \mathrm{uL}$ del sedimento a tubos de microcentrífuga estériles de $1,5 \mathrm{~mL}$ y luego se les agregó $360 \mathrm{uL}$ de buffer (buffer AL Qiagen).

Para determinar cuál es el procedimiento más eficiente para obtener ADN, se ensayaron tres métodos: congelacióndescongelación, congelación-ebullición (choque térmico) y batido en perlas de vidrio con buffers de lisis. Se demostró que, el método de choque térmico fue el más eficiente; por ello, las muestras se sometieron a dicho método. Primero, fueron congeladas a $-70{ }^{\circ} \mathrm{C}$ por diez minutos y sometidas a ebullición a $100{ }^{\circ} \mathrm{C}$ por dos minutos, luego se les dejó enfriar. Se les agregó $20 \mathrm{uL}$ de proteinasa $\mathrm{K}$ a una concentración de $20 \mathrm{mg} / \mathrm{mL}$ (Qiagen) y se les incubó por dos horas a $56^{\circ} \mathrm{C}$. La purificación del ADN se realizó con el Mini Kit QIAamp DNA (Qiagen) siguiendo el protocolo establecido. La concentración de ADN fue determinada mediante la medición de la absorción a $260 \mathrm{~nm}$, obteniendo una concentración mínima de 100 ng/mL ${ }^{(14)}$.

Para las reacciones de amplificación se utilizaron: Primers PCR: P1 (5' GAA AGG TCA GGT GTT CGA GTC A-3') y P2 (5' CTT GGT TGC GGG TTT AGG GAT T-3'), y sondas: sonda "alta", tRNA FL (5' TTC TTA TTT ATA TGC GGG TTG ATG TAA TAG TAA CA-3'), que contenía un marcador de fluoresceína en la terminación 3' y sonda «baja», tRNA LC (5`-AGA TGG CTC ATG ACC ATA ATA TTT AGG TGC p), que contenía un marcador LC Red 640 en la terminación 5'. La mezcla de PCR optimizada (20 uL), contenía 1 uL de cada primer a 0,5 uM, $1 \mathrm{uL}$ de cada sonda a 0,15 uM, 5uL de ADN molde, 9 uL de mastermix y 2 uL de agua PCR. Los 45 ciclos de amplificación incluyeron desnaturalización a $95{ }^{\circ} \mathrm{C}$ durante diez segundos, hibridación de los primers y sondas a $60{ }^{\circ} \mathrm{C}$ durante diez segundos, y extensión a $72{ }^{\circ} \mathrm{C}$ durante ocho segundos. Cuando las dos sondas fueron hibridadas a sus primers, la fluorescencia producida fue detectada y medida por el termociclador (PikoReal, Thermo Fisher Scientific) al final de la fase de hibridación de cada ciclo $^{(16)}$. La amplificación del ADN molde en las reacciones se midió usando el software Pico Real 2.2 del instrumento. En este método, el valor $C Q$ (ciclo de cuantificación) fue el punto de corte considerado por el equipo, a partir del cual, se consideraron las amplificaciones como positivas ${ }^{(15)}$.

\section{RESULTADOS}

\section{IDENTIFICACIÓN FENOTÍPICA DE ASPERGILLUS FUMIGATUS SENSU LATO}

Macroscópicamente, las 20 cepas sembradas en agar Czápeck con extracto de levadura e incubadas a $25{ }^{\circ} \mathrm{C}$, desarrollaron colonias de 35 a $70 \mathrm{~mm}$ de diámetro, de color verde azulado a verde grisáceo, con reverso incoloro o amarillo y a $37{ }^{\circ} \mathrm{C}$ desarrollaron colonias de 50 a $80 \mathrm{~mm}$ de diámetro, de color blanco cremosas y en algunos casos de aspecto algodonoso (Figura 1).

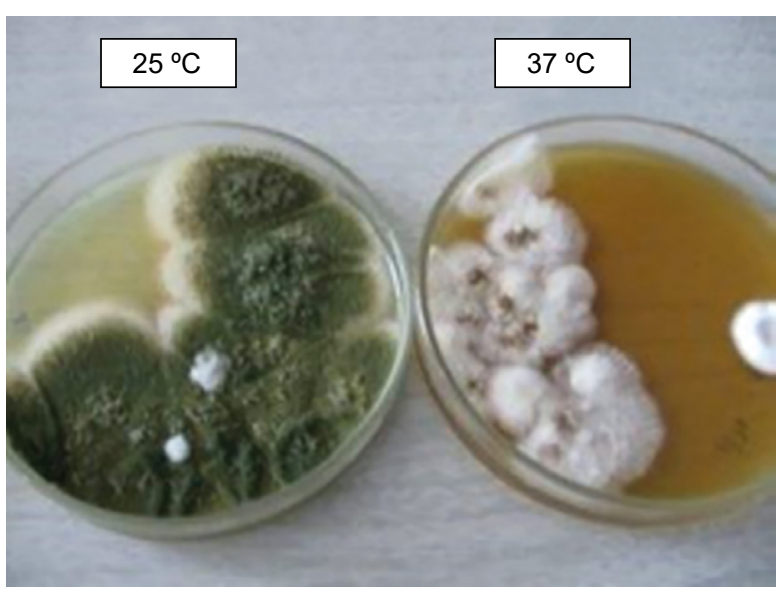

Figura 1. Colonias de Aspergillus fumigatus sensu lato, en agar Czápeck con extracto de levadura incubadas a $25^{\circ} \mathrm{C}$ y $37^{\circ} \mathrm{C}$.

Microscópicamente, tanto a $25^{\circ} \mathrm{C}$ como a $37^{\circ} \mathrm{C}$, presentaron las mismas características: cabezas aspergilares uniseriadas, vesículas piriformes con fiálides ocupando dos tercios de la vesícula. Conidios globosos y superficie lisa. Por lo que, el total de cepas corresponderían a Aspergillus fumigatus sensu lato (Figura 2).

\section{IDENTIFICACIÓN MOLECULAR DE Aspergillus fumigatus SENSU STRICTO POR PCR EN TIEMPO REAL}

Las curvas de amplificación de ADN por PCR en tiempo real (qPCR) muestran de manera exponencial las unidades relativas de fluorescencia (RFU) producidas a medida que avanzan los ciclos del qPCR, hasta llegar a su plateau en el ciclo 45. Cada línea coloreada representa una muestra distinta ya amplificada. Las curvas de fluorescencia obtenidas después del PCR en tiempo real muestran amplificación absoluta, estimada en RFU, consistentes en la mayoría de las muestras. Se considera positiva, cuando una curva está sobre los 42 ciclos. Mediante este procedimiento confirmamos la identificación de Aspergillus fumigatus sensu stricto en diez cepas de un total de 20 identificadas como $A$. fumigatus por métodos convencionales (Figura 3 y Tabla 1).

Se considera una curva positiva cuando se encuentra sobre los 42 ciclos. Cada línea coloreada representa una especie distinta ya amplificada. Se identificaron diez cepas de Aspergillus fumigatus sensu stricto, de 20 tipificadas como Aspergillus fumigatus sensu lato.

\section{DISCUSIÓN}

Los resultados obtenidos evidencian las limitaciones de los métodos convencionales de diagnóstico basados en las características morfológicas, utilizando técnicas de cultivo o histológicas para la identificación de Aspergillus fumigatus, aislados de muestras clínicas. En el Perú, han aumentado 


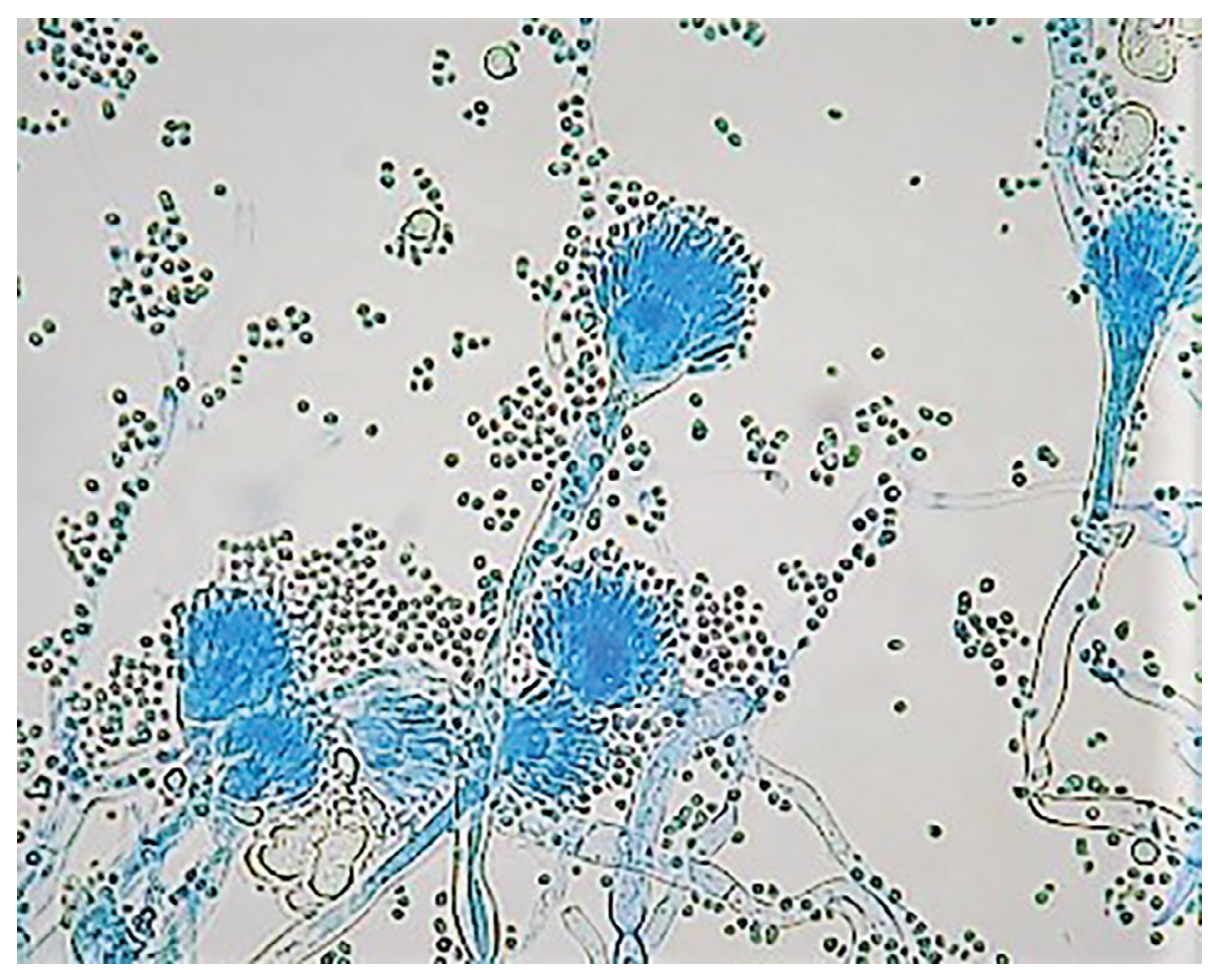

Figura 2. Aspergillus fumigatus sensu lato, microcultivo con azul de lactofenol (40 X).

los casos de Al producidos por A. fumigatus sensu lato resistentes al tratamiento, lo que motivó a utilizar la técnica de qPCR para reidentificar molecularmente las cepas aisladas. Logramos comprobar que sólo el $50 \%$ de las cepas evaluadas eran A. fumigatus sensu stricto, porcentaje significativamente discordante con estudios previos, en los cuales reportaron que entre $94 \%$ y $97 \%$ de las $\mathrm{Al}$ en humanos eran causados por Aspergillus fumigatus sensu stricto ${ }^{(8-16)}$.
Es importante mencionar que, en el ambiente también se encuentran cepas de $A$. fumigatus de la sección Fumigati que no corresponden a $A$. fumigatus sensu stricto; en un estudio de146 cepas de $A$. fumigatus sensu lato aislados de humanos, suelo y animales se determinó que el $4,1 \%$ no correspondían a $A$. fumigatus sensu stricto ${ }^{(3)}$ y en otro de 23 cepas de aspergillus de la sección Fumigati aisladas del suelo, el 60,8\% (14) no eran $A$. fumigatus sensu stricto ${ }^{(9)}$

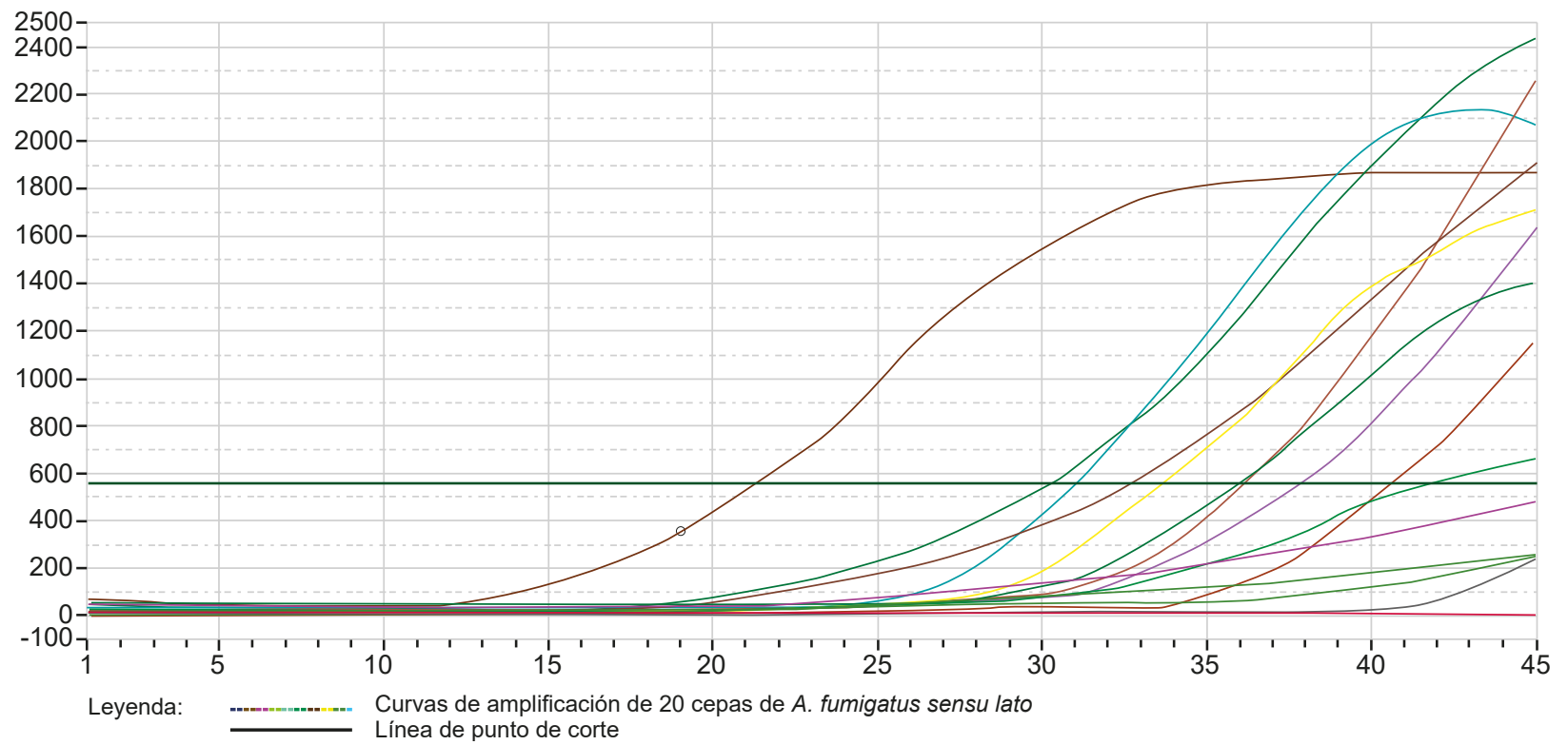

Figura 3. PCR en tiempo real (qPCR) de 20 cepas de Aspergillus fumigatus sensu lato. Curvas de amplificación de ADN 
Tabla 1. Amplificación por PCR en tiempo real de 20 cepas tipificadas fenotípicamente como Aspergillus fumigatus sensu lato.

\begin{tabular}{|c|c|c|c|}
\hline Número & $\begin{array}{l}\text { Código-cepas de Aspergillus } \\
\text { fumigatus sensu lato }\end{array}$ & $\begin{array}{c}\text { Muestras de pacientes con } \\
\text { diagnóstico de aspergilosis invasiva }\end{array}$ & $\begin{array}{c}\text { Amplificación por } \mathrm{qPCR}^{1} \text { para } \\
\text { Aspergillus fumigatus sensu stricto }\end{array}$ \\
\hline 1 & 8 & Biopsia tejido pulmonar & Positivo \\
\hline 2 & 19 & Esputo seriado & Negativo \\
\hline 3 & 28 & Aspirado bronquial & Negativo \\
\hline 4 & 33 & Esputo seriado & Negativo \\
\hline 5 & 47 & Esputo seriado & Negativo \\
\hline 6 & 67 & Esputo seriado & Positivo \\
\hline 7 & 135 & Esputo seriado & Positivo \\
\hline 8 & 130 & Biopsia tejido pulmonar & Positivo \\
\hline 9 & 128 & Esputo seriado & Positivo \\
\hline 10 & 126 & Esputo seriado & Positivo \\
\hline 11 & 85 & Aspirado bronquial & Positivo \\
\hline 12 & 79 & Esputo seriado & Negativo \\
\hline 13 & 301 & Esputo seriado & Negativo \\
\hline 14 & 302 & Esputo seriado & Negativo \\
\hline 15 & 303 & Biopsia tejido pulmonar & Positivo \\
\hline 16 & 304 & Biopsia tejido pulmonar & Positivo \\
\hline 17 & 642 & Esputo seriado & Positivo \\
\hline 18 & 270 & Esputo seriado & Negativo \\
\hline 19 & 14 & Esputo seriado & Negativo \\
\hline 20 & 83 & Esputo seriado & Negativo \\
\hline
\end{tabular}

qPCR: reacción en cadena de la polimerasa en tiempo real.

La discordancia entre nuestros resultados del estudio fenotípico ( $A$. fumigatus sensu lato) y del molecular $(A$. fumigatus sensu stricto) se debe a la presencia de cepas de aspergillus del grupo Fumigati que comparten características fenotípicas con A. fumigatus sensu stricto; resultado similar al reportado en estudios previos de $\mathrm{Al}$ realizados en otros países ${ }^{(17,18)}$. Entre las especies erróneamente identificadas como $A$. fumigatus se encuentran $A$. lentulus, $A$. viridinutans, Neorsartorya fischeri, $N$. pseudofischeri, $N$. udagawae y $N$. hiratsukae ${ }^{(4,5,7,17,19)}$. Los resultados obtenidos sugieren que algunas de estas especies estarían involucradas en los casos de Al en el Perú, contribuyendo a explicar la resistencia al tratamiento; y evidenciando la importancia del ambiente en el incremento de los casos.

La obtención de ADN fúngico es un reto en el diagnóstico molecular de las micosis invasivas, por ello, se evaluaron tres métodos ${ }^{(14,20)}$ y se seleccionó el de congelaciónebullición (choque térmico) ${ }^{(13,14,16)}$. La aplicación de este método, el tratamiento enzimático para la extracción de ADN fúngico y la posterior purificación mediante columnas de sílica-gel mostró ser el procedimiento más efectivo, porque permitió obtener mayores concentraciones de ADN. No obstante por limitaciones del laboratorio, no fue posible determinar molecularmente la especie de las cepas «no
A. fumigatus sensu stricto» ni evaluar la sensibilidad a los antifúngicos, de todas las cepas estudiadas.

De los resultados obtenidos, se concluye que al efectuarse el estudio molecular de cepas aisladas de casos de Al, identificadas fenotípicamente como Aspergillus fumigatus sensu lato, un elevado porcentaje de las mismas no corresponde a la especie Aspergillus fumigatus sensu stricto. Asimismo, el método de congelación-ebullición (choque térmico) sería el más efectivo para la obtención del ADN fúngico.

Contribuciones de autoría: VB, FV y GV diseñaron el estudio, realizaron una discusión crítica y se encargaron de la escritura final del manuscrito. SL y FV: establecieron el protocolo de trabajo, procesaron y analizaron los resultados. JGG se encargó del procesamiento del estudio fenotípico de las cepas y su análisis. $\mathrm{AU}$ proporcionó las cepas provenientes de aspergilosis invasivas y datos clínicos. AC y IS analizaron los resultados y brindaron aportes en la redacción del manuscrito. Todos los autores revisaron y aprobaron la versión final del manuscrito.

Fuentes de financiamiento: este trabajo fue financiado por el Vicerrectorado de Investigación y Posgrado (VRIP) de la Universidad Nacional Mayor de San Marcos.

Conflictos de interés: los autores declaran no tener ningún conflicto de interés. 


\section{REFERENCIAS BIBLIOGRÁFICAS}

1. Kwon-Chung KJ, Sugui JA. Aspergillus fumigatus-what makes the species a ubiquitous human fungal pathogen. PLoS pathogens. 2013;9(12): e1003743.

2. Klich MA, Pitt JI. A laboratory guide to common Aspergillus species and their teleomorphs. North Ryde, CSIRO Division of Food Processing;1988. pp $1-116$

3. Hong SB, Kim DH, Park IC, Choi YJ, Shin HD, Samson R. Re-identification of Aspergillus fumigatus sensu lato based on a new concept of species delimitation. Microbiol. 2010;48(5):607-615.

4. Balajee SA, Gribskov J, Brandt M, Ito J, Fothergill A, Marr KA. Mistaken identity: Neosartorya pseudofischeri and its anamorph masquerading as Aspergillus fumigatus. J Clin Microbiol. 2005;43(12):5996-5999.

5. Balajee SA, Gribskov JL, Hanley E, Nickle D, Marr KA. Aspergillus lentulus sp. nov., a new sibling species of $\mathrm{A}$. fumigatus. Eukaryot Cell. 2005;4(3):625-632.

6. Samson RA, Hong S, Peterson SW, Frisvad JC, Varga J. Polyphasic taxonomy of Aspergillus section Fumigati and its teleomorph Neosartorya. Stud Mycol. 2007;59:147-203.

7. Hong SB, Shin HD, Hong J, Frisvad JC, Nielsen PV, Varga J, Samson RA. New taxa of Neosartorya and Aspergillus in Aspergillus section Fumigati. Antonie van Leeuwenhoek. 2008;93(1-2):87-98.

8. Balajee SA, Kano R, Baddley JW, Moser SA, Alexander BD. Molecular identification of Aspergillus species collected for the transplant-associated infection surveillance network. J. Clin. Microbiol. 2009;4:3138-141. doi: 10.1128/ JCM.00162-06.
9. Giousiano GE, Piontelli E, Fernández MS, Mangiaterra ML, Cattana ME, Kocsubé S, Varga J. Biodiversity of species of Aspergillus Fumigati in semi-desert soils in Argentina. Rev. Argent. Microbol. 2017;247-254

10. Mellado E, Alcazar-Fuoli L, GarciaEffron G, Alastruey-Izquierdo A, CuencaEstrella M, Rodriguez-Tudela JL. New resistance mechanisms to azole drugs in Aspergillus fumigatus and emergence of antifungal drugs-resistant $\mathrm{A}$. fumigatus atypical strains. Medical Mycology. 2006;44(Supplement-1):S367-S371.

11. Howard SJ, Cerar D, Anderson MJ, et al. Frequency and evolution of azole resistance in Aspergillus fumigatus associated with treatment failure. Emerg Infect Dis. 2009;15(7):1068-1076.

12. Alcazar-Fuoli L, Mellado E, Alastruey-Izquierdo A, Cuenca-Estrella M, Rodriguez-Tudela JL. Aspergillus section Fumigati: antifungal susceptibility patterns and sequence-based identification. Antimicrob Agents Chemother. 2008;52(4):12441251

13. Alhambra A, Catalán M, Moragues MD, et al. Isolation of Aspergillus lentulus in Spain from a critically ill patient with chronic obstructive pulmonary disease. Rev Iberoam Micol. 2008;25:246-249.

14. Griffiths LJ, Anyim M, Doffman SR, Wilks M, Millar MR, Agrawal SG. Comparison of DNA extraction methods for Aspergillus fumigatus using real-time PCR. Journal of Medical microbiology. 2006;55(9):1187-1191.

15. Rantakokko-Jalava K, Laaksonen S, Issakainen J, Vauras J, Nikoskelainen J, Viljanen MK, et al. Semiquantitative detection by real-time PCR of Aspergillus fumigatus in bronchoalveolar lavage fluids and tissue biopsy specimens from patients with invasive aspergillosis. Journal of clinical microbiology. 2003;41(9):4304-4311.

16. Alastruey-Izquierdo A, Alcazar-Fuoli L, Cuenca -Estrella M. Antifungal susceotibility profile of cryptic species of Aspergillus. Mycopathologia. 2014;178:427433. doi: $10.1007 /$ s110-46-014-9775-z

17. Guarro J, Kallas EG, Godoy P, Karenina A, Gené J, Stchigel A, et al. Cerebral aspergillosis caused by Neosartorya hiratsukae,Brazil. Emerg Infect Dis. 2002;8(9):989-991.

18. Vinh DC, Shea YR, Sugui JA, ParrillaCastellar ER, Freeman AF, Campbell JW, et al. Invasive aspergillosis due to Neosartorya udagawae. Clin Infect Dis. 2009;49(1):102-111.

19. Gerber J, Chomicki J, Brandsberg JW, Jones R, Hammerman KJ. Pulmonary aspergillosis caused by Aspergillus fischeri var. spinosus: report of a case and value of serologic studies. Am. J. Clin. Pathol. 1973;60(6):861-866.

20. Fredricks DN, Smith C, Meier A. Comparison of six DNA extraction methods for recovery of fungal DNA as assessed by quantitative PCR. Journal of clinical microbiology. 2005;43(10): 5122-5128.

Correspondencia: Vilma R. Béjar Castillo

Dirección: Instituto de Medicina Tropical "Daniel A. Carrión" Facultad de Medicina, Germán Amézaga 375, Ciudad Universitaria. UNMSM. Cercado de Lima-Perú

Teléfono: 998018284

Correo electrónico:vbéjarc@unmsm.edu.pe

\section{Nuestros artículos se encuentran indizados en:}

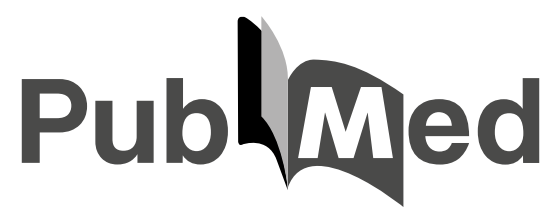

www.pubmed.gov 